\title{
High-mobility and low-power thin-film transistors based on multilayer $\mathrm{MoS}_{2}$ crystals
}

\author{
Sunkook Kim¹,2, Aniruddha Konar ${ }^{3}$, Wan-Sik Hwang ${ }^{3}$, Jong Hak Lee ${ }^{4}$, Jiyoul Lee', Jaehyun Yang4, \\ Changhoon Jung ${ }^{1}$, Hyoungsub Kim ${ }^{4}$, Ji-Beom Yoo ${ }^{4}$, Jae-Young Choi ${ }^{1}$, Yong Wan Jin ${ }^{1}$, Sang Yoon Lee ${ }^{1}$, \\ Debdeep Jena ${ }^{3}$, Woong Choi ${ }^{1,5} \&$ Kinam Kim ${ }^{1}$
}

Unlike graphene, the existence of bandgaps (1-2 eV) in the layered semiconductor molybdenum disulphide, combined with mobility enhancement by dielectric engineering, offers an attractive possibility of using single-layer molybdenum disulphide field-effect transistors in low-power switching devices. However, the complicated process of fabricating single-layer molybdenum disulphide with an additional high-k dielectric layer may significantly limit its compatibility with commercial fabrication. Here we show the first comprehensive investigation of processfriendly multilayer molybdenum disulphide field-effect transistors to demonstrate a compelling case for their applications in thin-film transistors. Our multilayer molybdenum disulphide field-effect transistors exhibited high mobilities ( $>100 \mathrm{~cm}^{2} \mathrm{~V}^{-1} \mathrm{~s}^{-1}$ ), near-ideal subthreshold swings ( $70 \mathrm{mV}$ per decade) and robust current saturation over a large voltage window. With simulations based on Shockley's long-channel transistor model and calculations of scattering mechanisms, these results provide potentially important implications in the fabrication of highresolution large-area displays and further scientific investigation of various physical properties expected in other layered semiconductors.

\footnotetext{
${ }^{1}$ Display Device Laboratory, Samsung Advanced Institute of Technology, Samsung Electronics, Yongin, Gyeonggi 446-712, South Korea. ${ }^{2}$ Department of Electronics and Radio Engineering, Kyung Hee University, Yongin, Gyeonggi 446-701, South Korea. ${ }^{3}$ Department of Electrical Engineering, University of Notre Dame, Notre Dame, Indiana 46556, USA. ${ }^{4}$ School of Advanced Materials Science and Engineering, Sungkyunkwan University, Suwon, Gyeonggi 440-746, South Korea. ${ }^{5}$ School of Advanced Materials Engineering, Kookmin University, Seoul 136-702 South Korea. Correspondence and requests for materials should be addressed to D.J. (email: djena@nd.edu) or to W.C. (email:woongchoi@kookmin.ac.kr).
} 
$\mathrm{T}$ he discovery of graphene opened the door to the exotic electronic, optical and mechanical properties of two-dimensional (2D) crystals ${ }^{1}$. Graphene has a conical Dirac spectrum of energy states without a bandgap and a linear dispersion. While these properties are the root of much of the novel electronic and optical phenomena of graphene, the gapless bandstructure also makes it unsuitable for conventional transistors for electronic switching. Similar to the storyline of the graphite and graphene family, transition metal dichalcogenides of the form $\mathrm{MX}_{2}$ where $\mathrm{M}=$ metal and $\mathrm{X}=\mathrm{S}$, Se or Te are emerging as highly attractive candidates for the study of fundamental physics in $2 \mathrm{D}$ and in layered (thin-film) structures. These materials form layered structures, where layers of covalently bonded $\mathrm{X}-\mathrm{M}-\mathrm{X}$ atoms are held together by Van der Waals interactions. But, because of the broken symmetry in the atomic basis, they can have bandgaps of $\sim 1 \mathrm{eV}$.

Among these layered semiconductors, recently, special emphasis has been given to single-layer (SL) $\mathrm{MoS}_{2}$ owing to its intriguing electrical and optical properties. While bulk $\mathrm{MoS}_{2}$ is usually an $n$-type semiconductor with an indirect bandgap $(\sim 1.3 \mathrm{eV})^{2}$ and carrier mobility in the $50-200 \mathrm{~cm}^{2} \mathrm{~V}^{-1} \mathrm{~s}^{-1}$ range at room temperature ${ }^{3}$, $\mathrm{SL} \mathrm{MoS}_{2}$ is found ${ }^{4}$ to have a direct bandgap of $\sim 1.8 \mathrm{eV}$. Field-effect transistors (FETs) using SL MoS exhibited high on/off ratios $\left(\sim 10^{8}\right)$ and low subthreshold swing (SS, $\sim 70 \mathrm{mV}$ per decade) ${ }^{5}$. The electron mobility of SL MoS ${ }_{2}$ FETs varied from $\sim 1 \mathrm{~cm}^{2} \mathrm{~V}^{-1} \mathrm{~s}^{-1}$ (in air/ $\mathrm{MoS}_{2} / \mathrm{SiO}_{2}$ structures) to $200 \mathrm{~cm}^{2} \mathrm{~V}^{-1} \mathrm{~s}^{-1}$ (in $\mathrm{HfO}_{2} / \mathrm{MoS}_{2} / \mathrm{SiO}_{2}$ structures) depending on dielectric environment. Thus, in addition to fundamental scientific interest, SL MoS 2 FETs can be an attractive alternative for electronic switches in the form of thin-film transistors (TFTs) for high-resolution liquid crystal displays and organic light-emitting diode (OLED) displays. These devices have a critical need for high field-effect mobility $\left(>30 \mathrm{~cm}^{2} \mathrm{~V}^{-1} \mathrm{~s}^{-1}\right)$, high on/off ratio $\left(>10^{3}\right)$, steep-subthreshold slopes for low power consumption, and electrical and optical reliabilities ${ }^{6}$. But, the synthesis of SL $\mathrm{MoS}_{2}$ followed by a deposition of an additional high- $k$ dielectric layer may not be well suited for commercial fabrication processes. Based on the physics of $\mathrm{MoS}_{2}$, we identify a number of reasons why multilayer $\mathrm{MoS}_{2}$ can be more attractive for TFT application than $\mathrm{SL} \mathrm{MoS}_{2}$. The density of states of multilayer $\mathrm{MoS}_{2}$ is three times that of SL $\mathrm{MoS}_{2}$, which will lead to considerably high drive currents in the ballistic limit. In long-channel TFTs, multiple conducting channels can be created by field effect in multilayer $\mathrm{MoS}_{2}$ for boosting the current drive of TFTs, similar to silicon-on-insulator MOSFETs.

However, multilayer $\mathrm{MoS}_{2}$ and corresponding dichalcogenide semiconductors have not been extensively studied for use in electronics. The characteristics in the few early reports ${ }^{7,8}$ are not vastly competitive with current TFT technologies. Therefore, in this work, we explore the case of multilayer $\mathrm{MoS}_{2}$ FETs further and show that it offers a compelling case for applications in TFTs. We report the first demonstration of multilayer $\mathrm{MoS}_{2}$ FETs with single back-gated insulator of 50 -nm-thick $\mathrm{Al}_{2} \mathrm{O}_{3}$ by atomic layer deposition (ALD) achieving high room-temperature mobilites $\left(>100 \mathrm{~cm}^{2} \mathrm{~V}^{-1} \mathrm{~s}^{-1}\right)$ and very low SS $(\sim 70 \mathrm{mV}$ per decade), which suggest that, with a proper use of a dielectric substrate, the mobility and the SS in multilayer $\mathrm{MoS}_{2}$ FETs may reach near the intrinsic limits at room temperature. We also demonstrate robust current saturation over a large voltage window. This is an important step towards real applications as TFTs in OLED displays are operated in the saturation region of drain current. Such saturation is elusive in FETs based on nanomaterials owing to poor electrostatic control, or exists in a very small voltage window in graphene owing to the lack of a bandgap 9 . We describe the experimental results with comprehensive simulations based on Shockley's long-channel transistor model and calculations of scattering mechanisms, and further suggest future directions to improve mobility in layered semiconductors.

\section{Results}

Device fabrication. TFTs based on multilayer $\mathrm{MoS}_{2}$ were fabricated with the architecture shown in Fig. 1a. After multilayer $\mathrm{MoS}_{2}$ flakes were mechanically exfoliated from bulk $\mathrm{MoS}_{2}$ crystals and transferred on $\mathrm{ALD}-\mathrm{Al}_{2} \mathrm{O}_{3}$-covered $\mathrm{Si}$ substrates, electrical contacts of $\mathrm{Au} / \mathrm{Ti}$ were patterned on top of $\mathrm{MoS}_{2}$ flakes as described in the Methods section. Optical micrograph of a completed device is shown is Fig. 1b. The thickness of $\mathrm{MoS}_{2}$ channels measured by atomic force microscope (AFM) was in the range of $20-80 \mathrm{~nm}$. Between the two stacking polytypes that are observed in bulk $\mathrm{MoS}_{2}$ crystals (hexagonal $2 \mathrm{H}$ and rhombohedral $3 \mathrm{R}$ ) ${ }^{10}$, crystal orientation mapping by electron backscatter diffraction indicated only $2 \mathrm{H}$ $\mathrm{MoS}_{2}$ within the measured devices as shown in Fig. 1c. Subsequent inverse pole figure in Fig. 1c confirmed that the $\mathrm{MoS}_{2}$ channels are $\langle 0001\rangle$-oriented single crystals. Raman spectra of $\mathrm{MoS}_{2}$ channels were almost identical with those of bulk single crystals as shown in Fig. 1d, and no noticeable difference in Raman shifts of $\mathrm{MoS}_{2}$ channels were found among measured devices, indicating minimal structural modifications.

Electronic properties. Figure 2 shows the measured device characteristics of a multilayer $\mathrm{MoS}_{2}$ TFT. The thickness of the $\mathrm{MoS}_{2}$ layer is $t_{\mathrm{ch}} \sim 30 \mathrm{~nm}$, and the back gate oxide thickness is $t_{\mathrm{ox}} \sim 50 \mathrm{~nm}$ (Fig. 2a). Figure $2 \mathrm{~b}$ shows the major features observed: the $n$-type nature of the $\mathrm{MoS}_{2}$ layer as indicated by it turning on at positive gate biases owing to accumulation of electrons, and a window of gate biases where the device stays off (depletion). This feature was observed in all measured devices, but a fraction of the devices also showed a recovery of the current at large negative gate biases as in Fig. 2b, which is a clear indication of an inversion channel. The inversion layer formation is described further in Supplementary Fig. S4 and in the Supplementary methods. We note that the gate capacitance in this geometry is $\sim 20$ times higher than a recent report ${ }^{7}$. As a result, while exhibiting the high on/off ratio expected of a semiconductor with a bandgap of $1.3 \mathrm{eV}$, a sharp SS ( $\sim 80 \mathrm{mV}$ per decade for Fig. $2,70 \mathrm{mV}$ per decade for some devices) is also measured at room temperature in deep depletion. In a typical field-effect geometry, the subthreshold slope is given by ${ }^{11} \mathrm{SS}=\left(1+\left(C_{\mathrm{S}}+C_{\mathrm{it}}\right) / C_{\mathrm{ox}}\right) \mathrm{kT} / \mathrm{q} \ln 10$, where $C_{\mathrm{S}}$ is the capacitance in the $\mathrm{MoS}_{2}$ conducting channel, $C_{\mathrm{it}}=q D_{\mathrm{it}}$ is the capacitance owing to interface traps of density $D_{\mathrm{it}}$ and $C_{\mathrm{OX}}=\varepsilon_{\mathrm{OX}} / t_{\mathrm{ox}}$ is the oxide capacitance. Based on this model, $\left(C_{\mathrm{S}}+C_{\mathrm{it}}\right) \sim C_{\mathrm{ox}} / 3$. As the semiconductor capacitance is negligible in the deep-subthreshold region, the interface trap density is $D_{\mathrm{it}} \sim 2.6 \times 10^{11} \mathrm{eV}^{-1} \mathrm{~cm}^{-2}$, a very low value indeed ${ }^{12}$. The SS value was measured to be $\sim 24 \mathrm{mV}$ per decade at $77 \mathrm{~K}$, indicating $D_{\text {it }}$ does not vary with temperature. We note that similar to the recent report ${ }^{5}$ for monolayer $\mathrm{MoS}_{2}$, the subthreshold slope (and $D_{\mathrm{it}}$ ) of multilayer $\mathrm{MoS}_{2}$ TFTs is also exceptionally low, comparable to state-of-the-art silicon transistors. But it is obtained without the decades of processing improvement that was necessary to reduce interface trap densities between the dielectric insulator and silicon. Currently III-V semiconductors are facing the same challenge ${ }^{13}$. This is because of the layered nature of the semiconductor-for 2D crystals and their stacks, there are no outof-plane broken bonds, and thus the interface traps are expected to be in the dielectrics and materials placed in contact with them, not in the semiconductor itself. This is a major advantage of $\mathrm{MoS}_{2}$ for TFT applications.

Current saturation. The second major boost is seen in Fig. 2c. The drain current is observed to saturate at high drain biases for all gate voltages. The saturation occurs over a wide drain voltage window (unlike graphene). This is the first observation of a robust current saturation in a layered semiconductor composed of $2 \mathrm{D}$ crystals. Current saturation in transistors is an important feature towards real applications as the TFTs in OLED displays are operated in the saturation region. Like in a long-channel transistor made of 
a

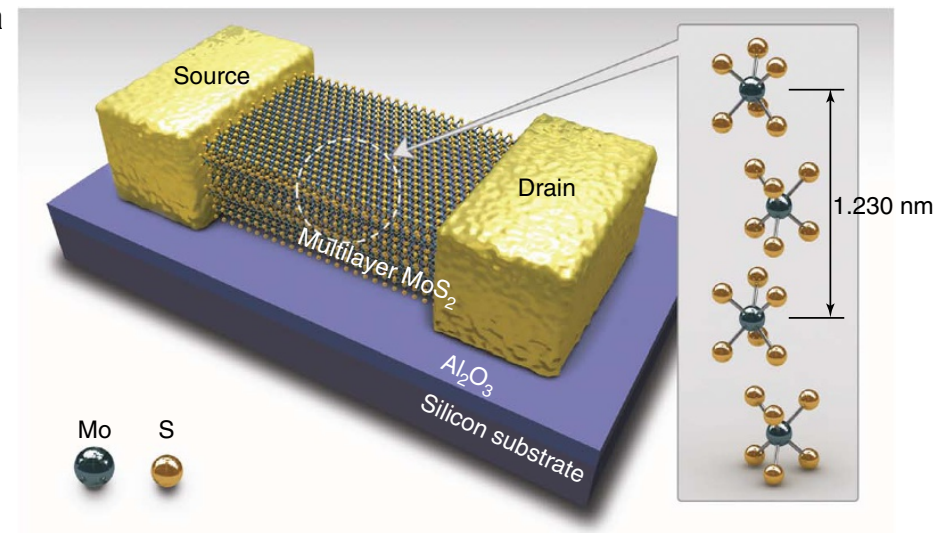

b
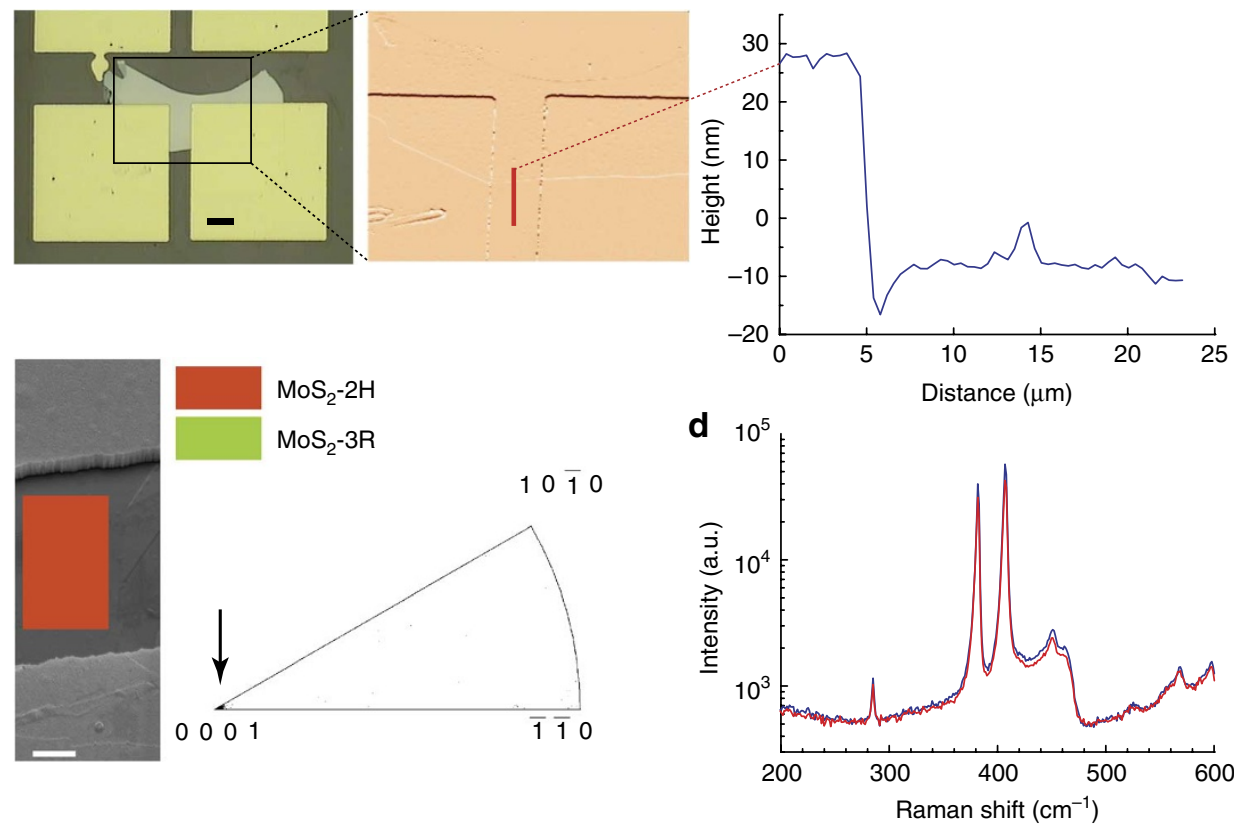

Figure 1 | MoS $_{\mathbf{2}}$ TFT device and its structural properties. (a) Schematic perspective view of a MoS 2 TFT with a multilayer MoS 2 crystal. (b) Optical and AFM image of a device deposited on top of a silicon substrate with a $50-\mathrm{nm}$-thick $\mathrm{Al}_{2} \mathrm{O}_{3}$ layer. The scale bar is $20 \mu \mathrm{m}$. Also shown is a cross-sectional plot along the red line in AFM image. (c) Scanning electron microscope image of the $\mathrm{MoS}_{2}$ channel with a crystal orientation mapping. The scale bar is $3 \mu \mathrm{m}$. Also displayed is a partial inverse pole figure indicating a $\langle 0001\rangle$-oriented single crystal. (d) Raman spectroscopy measurements on a bulk single crystal (blue) and a transistor channel (red).

a covalent semiconductor, the saturation of current occurs in the $\mathrm{MoS}_{2}$ TFT owing to pinch-off of the conducting channel at the drain side as the gate-drain diode becomes reverse-biased at high $V_{\mathrm{DS}}$. As graphene has zero bandgap, instead of pinch-off, the drain side of the conducting channel becomes p-type at high drain bias ${ }^{9}$, restricting current saturation and current modulation to a very small window, if at all. The bandgap of $\mathrm{MoS}_{2}$ makes both current modulation and saturation robust, as borne out by Fig. 2b,c.

The saturation of current observed here is quantitatively understood based on a long-channel device model based on surface potential. Poisson equation is solved to determine the surface potential at the $\mathrm{MoS}_{2}$ /ALD oxide interface as a function of the gate and the drain bias voltages. Then, the Shockley model of transistor performance is used to calculate the current. The only unknown input parameters to the model are the carrier mobility, the doping density and the contact resistance. At high positive gate biases, the channel is flooded with accumulated carriers and is highly conductive, and the contact resistances limit the current. This helps us to deduce the contact resistance to be $\sim 17 \mathrm{k} \Omega$ as described in Supplementary Fig. S1 and in the Supplementary methods. This value is rather high, and reducing it by an order of magnitude is necessary for the future.

Thus, the mobility and the doping density remain as the unknown parameters. In Fig. 2d, using a mobility of $100 \mathrm{~cm}^{2} \mathrm{~V}^{-1} \mathrm{~s}^{-1}$ and a doping density of $N_{\mathrm{D}^{\sim}} 10^{16} \mathrm{~cm}^{-3}$, an excellent fit to the measured device characteristics is obtained. We assert that these values are not fortuitous, as borne out by complementary capacitance-voltage and field-effect mobility measurements, which are described next. The device model for TFTs provides insight into the performance of $2 \mathrm{D}$ layered semiconductor devices, and thus is a powerful tool to extract physical parameters of the material.

Accumulation and inversion channels. At large negative gate biases, the drain current recovers as seen in Fig. 2b, indicating the formation of an inversion channel (formation of a hole gas in a $n$-type semiconductor). However, the source/drain contacts are formed to 

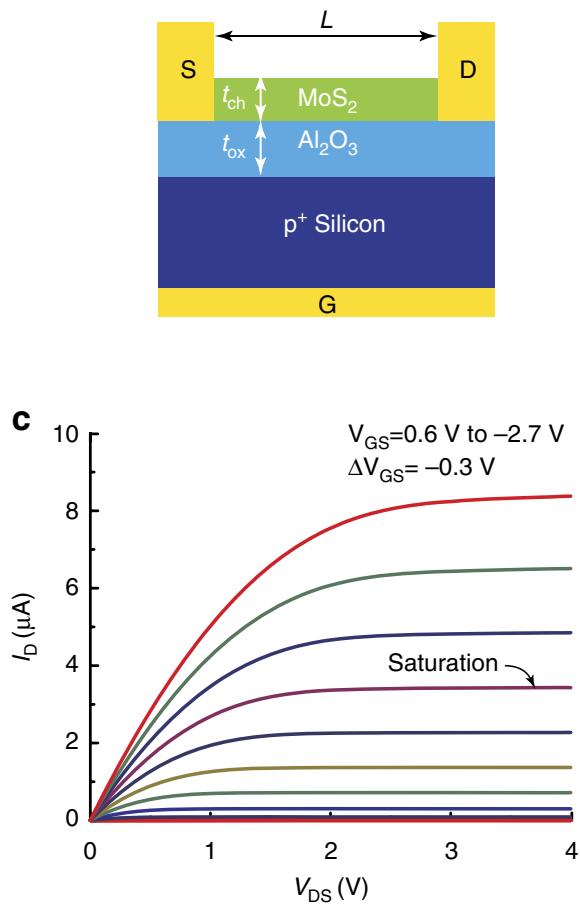

b

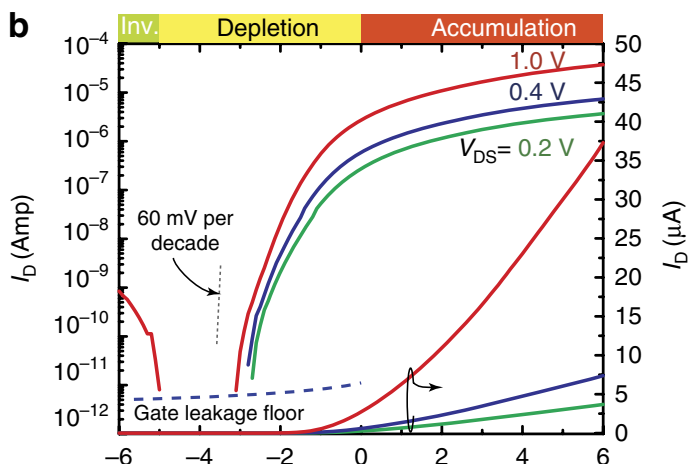

d

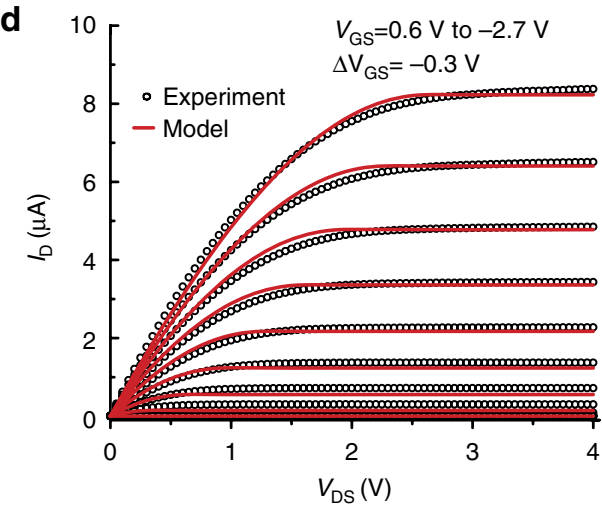

Figure 2 | Device geometry and electronic properties of $\mathbf{M o S}_{\mathbf{2}}$ TFTs. (a) The device geometry of a back-gated MoS 2 TFT. The TFT device was measured at $T=300 \mathrm{~K}$. The device geometries are: $W / L=4 / 7 \mu \mathrm{m}, t_{\mathrm{ox}}=50 \mathrm{~nm}$ and $t_{\mathrm{ch}}=30 \mathrm{~nm}$. (b) Drain current versus back gate bias showing $\sim 10^{6}$ on $/ \mathrm{off}_{\mathrm{ratio}}$ and $\sim 80 \mathrm{mV}$ per decade subthreshold slope. (c) Drain current versus drain bias showing current saturation. (d) Same as (c), including a long-channel model (red lines) showing excellent agreement between the TFT model and measured device behaviour.

the conduction band, and therefore there exists a large barrier for conduction through a p-type inversion channel. To explore these features quantitatively, capacitance-voltage measurements were performed, and the measured device characteristics were compared with energy-band diagram-based models.

Figure $3 \mathrm{a}-\mathrm{c}$ show the calculated energy band diagrams for the $\mathrm{MoS}_{2}$ TFT structure for various gate bias conditions. For the calculation, we self-consistently solve Poisson and Schrödinger equations in the effective-mass approximation. The bandgap of SL $\mathrm{MoS}_{2}$ occurs at the $K$-points in the hexagonal k-space lattice ${ }^{4}$, implying a valley degeneracy of $g_{\mathrm{v}}=2$, similar to graphene. On the other hand, the conduction band minimum of multilayer $\mathrm{MoS}_{2}$ moves to a lower symmetry point in the $k$-space along the $\Gamma-\mathrm{K}$ line. This results in a higher valley degeneracy $\left(g_{\mathrm{v}}=6\right.$ for the $\Gamma-\mathrm{K}$ line) than SL $\mathrm{MoS}_{2}$, effectively tripling the density of states, implying higher carrier densities and higher currents in the ballistic limit ${ }^{14}$. The net drive current for a given voltage is a product of the carrier density and the velocity. Thus, in addition to high velocity, a high density of states (DOS) is equally attractive for attaining high speed. Driven by higher valley degeneracy, multilayer $\mathrm{MoS}_{2}$ has the potential for considerably higher current drives than SL $\mathrm{MoS}_{2}$ in the ultrascaled limit, and high charge densities have recently been reported ${ }^{15}$. Even in the long-channel structure, thin-film $\mathrm{MoS}_{2}$ can take advantage of its multilayer nature. It can provide multiple conducting channels for boosting the current drive by using double gates, similar to silicon-on-insulator MOSFETs. The semiconducting material properties of multilayer $\mathrm{MoS}_{2}$ such as the conduction and valence band offsets with $\mathrm{Si}$ and $\mathrm{ALD} \mathrm{Al}_{2} \mathrm{O}_{3}$, the valley degeneracy, band-edge effective masses, dielectric constant and bandgap were used in the calculation. The values are provided in Supplementary Table S1 and Fig. S6. The model indicates that owing to the work-function difference between $\mathrm{MoS}_{2}$ and $\mathrm{p}^{+} \mathrm{Si}$, the thin $\mathrm{MoS}_{2}$ layer is initially depleted of mobile carriers. Upon application of positive bias on the Si gate, a 2D electron gas in the $\mathrm{MoS}_{2}$ layers closest to the ALD $\mathrm{Al}_{2} \mathrm{O}_{3}$ forms. This accumulation channel conducts current between the source and the drain. Figure $3 \mathrm{~d}$ shows the accumulation carrier density profile in more detail. Most of the carriers are electrostatically confined close to the $\mathrm{MoS}_{2}$ /ALD interface, similar to the case in a Si MOSFET. Thus, the 'quantum capacitance', which dictates the voltage drop in the semiconductor to sustain the conducting charge $C_{S} \sim \varepsilon_{S} /\langle z\rangle$ is large as the centroid of the charge distribution $\langle z\rangle$ is $\sim 1-2 \mathrm{~nm}$ from the interface.

Capacitance measurements. Figure $3 e$ shows the measured twoterminal capacitance as a function of the voltage between an ohmic contact pad to the $\mathrm{MoS}_{2}$ and the back gate. Several interesting features are evident from the measurement. As the pads are large as seen in Fig. 1b, they form a parasitic pad capacitance, which sets the floor of the measured value $(\sim 12 \mathrm{pF})$. When the $\mathrm{MoS}_{2}$ layer is depleted of mobile carriers, the measured capacitance is this pad capacitance. As positive gate biases are applied, the formation of an electron accumulation layer in the $\mathrm{MoS}_{2}$ results in an increase in the capacitance. The electron accumulation layer also electrically connects the two ohmic pads, effectively doubling the parasitic pad capacitance-this is exactly what is measured. In Supplementary Fig. S2 and the Supplementary methods, we describe this effect in more detail, and also include the capacitance-voltage measurements of a $\mathrm{MoS}_{2}$ flake in contact with just one metal pad in Supplementary Fig. S3, which confirms the above analysis. The capacitance of the $\mathrm{MoS}_{2}$ layer alone $\left(C_{\mathrm{MoS}_{2}}\right)$ is dependent on its doping density. The calculated values of $C_{\mathrm{MoS}_{2}}$ for three different doping densities are shown in Fig. 3e, from which it is concluded that the 

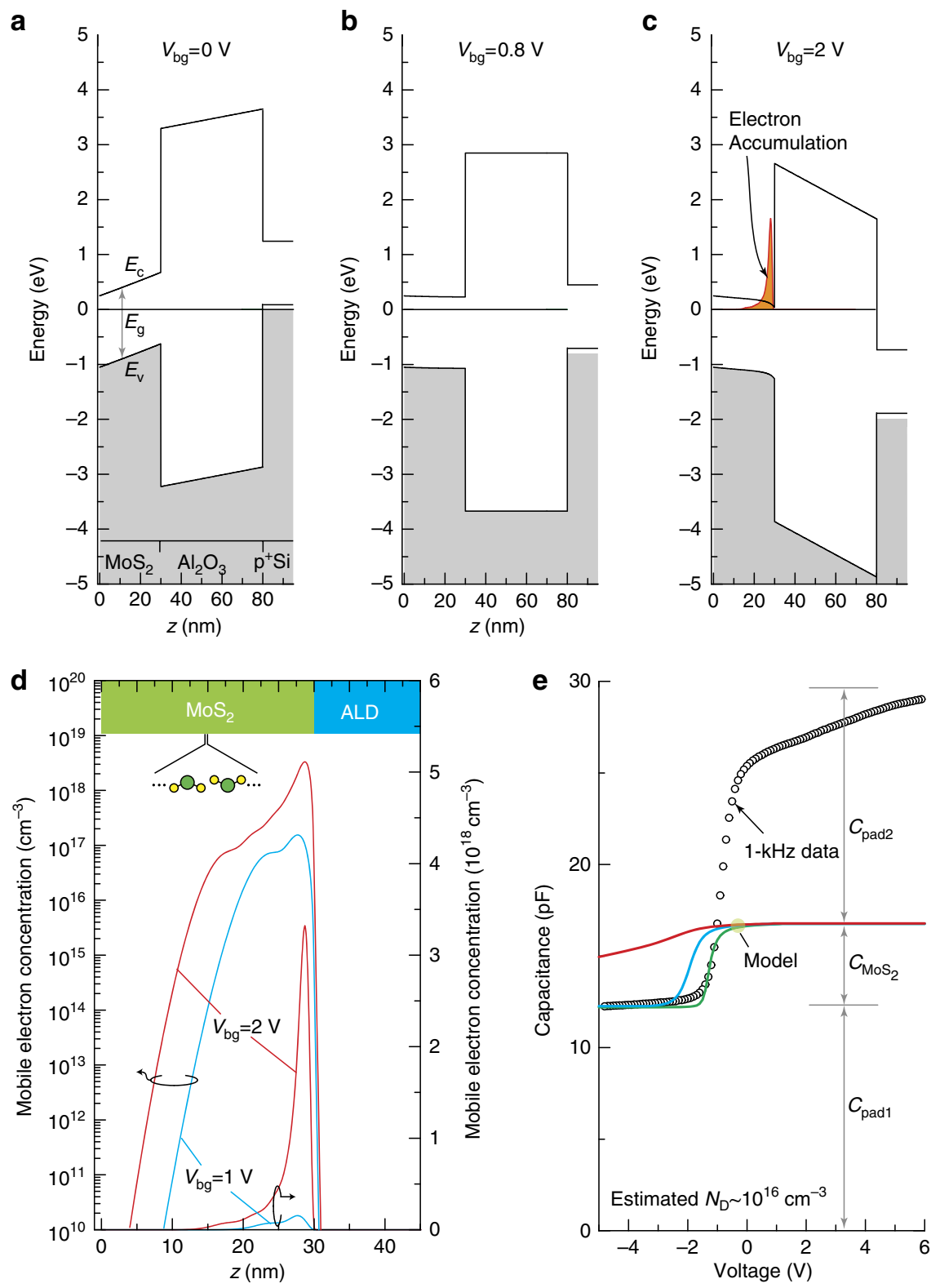

Figure 3 | Energy band diagrams, carrier distributions and measured capacitance-voltage profile of the $\mathbf{M o S}_{\mathbf{2}}$ TFT device. (a-c) Energy band diagrams of the $\mathrm{MoS}_{2} / \mathrm{Al}_{2} \mathrm{O}_{3} / \mathrm{p}^{+} \mathrm{Si}$ device under various bias conditions. The band offsets and physical parameters relevant for the calculation are described in the Supplementary methods. The self-consistent Schrödinger-Poisson calculation shows that at large positive gate bias, a 2D electron gas is formed at the $\mathrm{MoS}_{2} / \mathrm{Al}_{2} \mathrm{O}_{3}$ interface. (d) The 2D electron gas that forms the conductive channel is shown in an enlarged scale. Most conduction occurs by electrons accumulated in a few layers at the $\mathrm{MoS}_{2} / \mathrm{Al}_{2} \mathrm{O}_{3}$ interface. With increasing bias, the centroid of the electron distribution shifts closer to the interface. This indicates that the 'quantum' capacitance in the semiconductor increases with positive gate bias. (e) Measured capacitance-voltage curves of the MoS $2 /$ $\mathrm{Al}_{2} \mathrm{O}_{3}$ /back-gate capacitors (circles). The solid lines show the calculated capacitance for three different doping densities (red: $10^{18}$, blue: $10^{17}$ and green: $10^{16} \mathrm{~cm}^{-3}$ ), not including parasitic pad capacitances. The slope indicates a doping density close to $10^{16} \mathrm{~cm}^{-3}$.

unintentional doping density in the measured $\mathrm{MoS}_{2}$ layer is of the order of $N_{\mathrm{D}^{\sim}} 10^{16} \mathrm{~cm}^{-3}$. We note that this is a low doping level, and can vary between naturally occurring samples not grown by controlled means. The extracted doping density has a direct impact on impurity scattering and carrier mobility, which is what was investigated next.

Charge transport and scattering. The field-effect mobility of $\mathrm{MoS}_{2}$ TFTs was extracted from the $I_{\mathrm{D}}-V_{\mathrm{GS}}$ curves of Fig. $2 \mathrm{~b}$, and the corresponding measurements for temperatures $77-300 \mathrm{~K}$. The mobility values are extracted in the linear region at $V_{\mathrm{GS}}=2 \mathrm{~V}$ or equivalently at a carrier density $n=C_{\mathrm{ox}}\left(V_{\mathrm{GS}}-V_{\mathrm{T}}\right) \approx 1.6 \times 10^{12} \mathrm{~cm}^{-2}$. The intrinsic carrier mobility (mobility without the effect of the contact resistance $R_{\mathrm{c}}$ ) was calculated using an equivalent circuit as shown in Fig. $4 \mathrm{c}$ and described further in Supplementary Fig. S5 and the Supplementary methods, and are shown as blank circles in Fig. 4a. Also shown are data reported from Fivaz and Mooser's work ${ }^{3}$ from 1967. The values are very similar. We note here that Fivaz and Mooser used $\mathrm{MoS}_{2}$ crystals grown by transport reaction as opposed to the naturally occurring $\mathrm{MoS}_{2}$ used in this work, which bodes well for large-area growth for practical TFT applications. The decrease in mobility with temperature is a typical signature of diffusive band transport, as opposed to activated (hopping) transport. If there were significant Schottky barrier heights, the mobility would appear to 


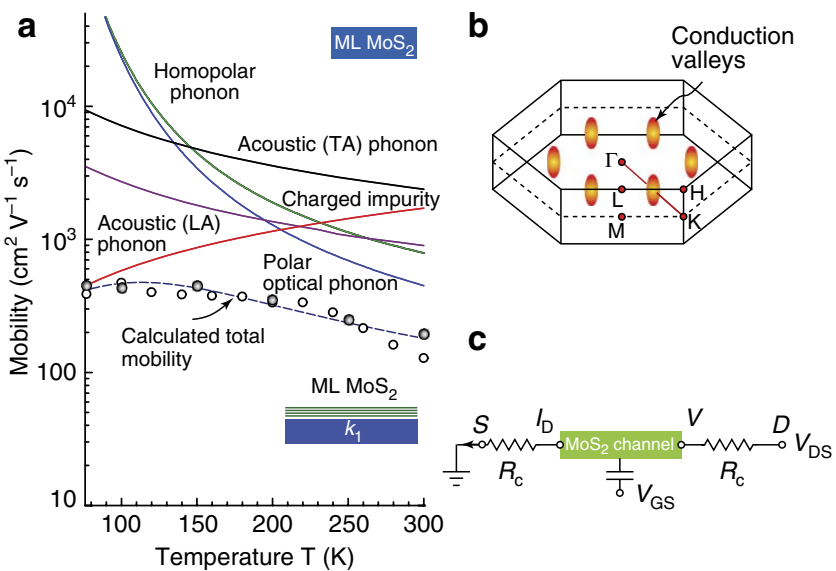

Figure 4 | Charge transport properties of the multilayer $\mathrm{MoS}_{\mathbf{2}}$ channel.

(a) Measured temperature-dependent field-effect mobility of $\mathrm{MoS}_{2}$ TFTs. The open circles are data measured in this work, and the filled circles are data from ref. 3. From the theoretical transport model, the electron mobility (dashed line) is limited by impurity scattering (red line) at low temperatures. At room temperature, the mobility is limited by the combined effect of the homopolar (out-of-plane) phonon (green line) and the polar-optical phonon (blue) scattering. Details of these scattering mechanisms are described in the Supplementary methods. (b) The hexagonal Brillioun zone of multilayer $\mathrm{MoS}_{2}$ with the high symmetry points and six equivalent conduction valleys. (c) An equivalent circuit model for the $\mathrm{MoS}_{2}$ TFT including the effect of the contact resistance $R_{\mathrm{c}}$.

increase with temperature. This can lead to erroneous conclusions on the nature of charge transport (that is, activated versus band transport). To avoid such confusion, samples that exhibited ohmic contacts over the entire temperature range were carefully chosen for mobility extraction.

To explain the temperature-dependent carrier mobility, the semiclassical Boltzmann transport equation under the relaxation time approximation is used. The model is described in detail in the Supplementary methods. A typical characteristic of layered structures (such as $\mathrm{MoS}_{2}$ ) is that the carriers move independently in each layer. Neglecting the vanishing interlayer interaction, the energy dispersion of carriers becomes ${ }^{16} E(k)=\hbar^{2}\left(k_{x}^{2}+k_{y}^{2}\right) / 2 m^{*}$, where $\mathbf{k}=\left(k_{x}, k_{y}\right)$ is the $2 \mathrm{D}$ wave vector of carriers and $m^{*}$ is the in-plane effective mass. The corresponding $2 \mathrm{D}$ density of states is $g_{2 d}=g_{\mathrm{v}} m^{*} / \pi \hbar^{2}$, where $\hbar$ is the reduced Planck constant and $g_{\mathrm{v}}$ is the valley degeneracy. Recent experiments and models indicate the conduction band minima to be along the $\Gamma-\mathrm{K}$ line ${ }^{17}$ of the Brillouin zone, as indicated in Fig. 4b. The sixfold symmetry of this point leads to a valley degeneracy of $g_{\mathrm{v}}=6$. We have used this value for transport calculations, consistent with the energy band diagram and capacitance calculations of Fig. 3. Carrier scattering from (a) ionized impurities, (b) acoustic phonons, (c) in-plane polar optical phonons and (d) out-of-plane lattice vibrations (homopolar phonons) are taken into account to explain the transport measurements. A 2D ionized impurity scattering model is invoked where carriers scatter from a sheet of impurities located at the surface of the $\mathrm{MoS}_{2}$ layers. Coupling of carrier with both longitudinal acoustic (LA) phonons and transverse acoustic (TA) phonons are taken into account under the deformation potential approximation. The energies of optical phonons in bulk $\mathrm{MoS}_{2}$ are in the frequency range of $400-500 \mathrm{~cm}^{-1}$ $(50-60 \mathrm{meV})^{19}$ as also measured in Fig. 1d. Electron-polar optical phonon scattering is described by the Fröhlich interaction ${ }^{20}$ with a static dielectric constant $\varepsilon_{0}=7.6$ and optical dielectric constant ${ }^{21}$ $\varepsilon_{\infty}=7.0$ with phonon energy $\hbar \omega_{\mathrm{op}}=49 \mathrm{meV}$ for the $E_{2 g}^{1}$ mode.
For homopolar phonon modes, the sulphur atoms of opposite planes vibrate out of phase $\left(A_{1 g}\right.$ mode) and the corresponding phonon energy is ${ }^{19,22} \hbar \omega_{\mathrm{hp}}=52 \mathrm{meV}$.

The resultant mobility is calculated using Mathiessen's rule $\mu^{-1}=\mu_{\mathrm{imp}}^{-1}+\mu_{\mathrm{LA}}^{-1}+\mu_{\mathrm{TA}}^{-1}+\mu_{\mathrm{op}}^{-1}+\mu_{\mathrm{hp}}^{-1}$. The calculated mobility associated with the individual scattering mechanisms as well as the resultant mobility is shown in Fig. 4a along with the measured data. At low temperatures, the mobility is limited by ionized impurity scattering. At room temperature, the mobility decreases by enhanced optical phonon and acoustic phonon scattering. A reasonably good match is found between calculated and experimentally measured mobility (at low temperatures) for a choice of impurity sheet density $n_{\mathrm{imp}} \sim 1.8 \times 10^{10} \mathrm{~cm}^{-2}$, which corresponds to a volume density of $\sim 3 \times 10^{15} \mathrm{~cm}^{-3}$. This value is comparable to the background doping density for unintentionally doped bulk $\mathrm{MoS}_{2}$, and consistent with the value estimated from the capacitance-voltage measurements in Fig. 3e.

A large electron effective mass and the strong optical phonon (out-of-plane and polar phonon) scattering set an upper bound on the mobility in multilayer $\mathrm{MoS}_{2}$. We note here that the accuracy of the calculation is subject to the uncertainty in the electronphonon coupling coefficients and the bandstructure parameters used in the model. However, these parameters are not expected to be vastly different from those assumed. Higher mobilities could potentially be achieved by intercalation of $\mathrm{MoS}_{2}$ layers (similar to recently demonstrated encapsulations of silicon carbide crystals in graphite enclosure ${ }^{23}$ ). By sandwiching SL $\mathrm{MoS}_{2}$ between two dielectric layers, the out-of-plane phonon vibrations can be suppressed. If the homopolar phonon mode is damped, ionized impurity scattering and in-plane polar optical phonon scattering determine the charge transport. Strain effects can also potentially be used to deform the bandstructure, leading to lowering of the electron effective mass and improvement in mobility. Electron mobility in $\mathrm{MoS}_{2}$ can be further expected to improve as the growth and processing methods improve, leading to fewer impurities. Moreover, charged impurity scattering in these devices can be damped using high $k$ dielectrics (dielectric engineering) ${ }^{24-26}$. Effects such as remote phonon scattering could limit this improvement ${ }^{27}$. As the current experimental values are far below limits expected of remote phonon scattering, there is ample room for improvement.

\section{Discussion}

The mobility measured for multilayer $\mathrm{MoS}_{2}$ already exceeds most competing semiconductor materials for large-area TFTs by orders of magnitude. The values may be further improved by proper dielectric choices to near the intrinsic phonon limits. When combined with the large current modulation, the low subthreshold slope, and robust current saturation, multilayer $\mathrm{MoS}_{2}$ makes a compelling case for TFT applications. All these properties are achieved in a back-gated structure, without the need for an additional dielectric layer on top, which is highly attractive for TFT implementation. The first demonstration of these attractive properties, combined with the comprehensive modelling of the behaviour, is expected to move multilayer $\mathrm{MoS}_{2}$ towards real applications. The multilayer structure is easier to achieve over large areas by chemical vapour deposition or allied techniques, which are well suited for largearea applications. Looking beyond $\mathrm{MoS}_{2}$, other transition metal dichalcogenides can offer competitive or complementary features. In addition to technological applications, conduction band electron states in these layered semiconductors have contributions from d orbitals, quite unlike traditional group-IV and III-V semiconductors and carbon nanomaterials, where chemical bonding is restricted to $\mathrm{s}$ and $\mathrm{p}$ orbitals. Thus, a rich range of physical phenomena that depend on d orbitals, such as magnetism, correlated-electron effects and superconductivity, can be expected in these materials. Such features can possibly be integrated with the 
semiconducting properties demonstrated here seamlessly, as there are no out-of-plane bonds to be broken.

\section{Methods}

Device fabrication. An amorphous $\mathrm{Al}_{2} \mathrm{O}_{3}$ dielectric layer of $\sim 50 \mathrm{~nm}$ in thickness was deposited on a highly doped p-type Si wafer (resistivity $<5 \times 10^{-3} \Omega \mathrm{cm}$ ) by ALD process using trimethylaluminum (TMA, UP Chemical Co. Ltd., South Korea) and $\mathrm{H}_{2} \mathrm{O}$ as a precursor and a reactant, respectively. The deposition temperature was maintained at $300^{\circ} \mathrm{C}$ and the gas injection schedule for one cycle of deposition were $0.5 / 10 / 1.5 / 15$ seconds for the TMA $/ \mathrm{N}_{2} / \mathrm{H}_{2} \mathrm{O} / \mathrm{N}_{2}$ gases. Multilayer $\mathrm{MoS}_{2}$ flakes were mechanically exfoliated from bulk $\mathrm{MoS}_{2}$ crystals (SPI Supplies, USA) and transferred on the substrate. Electrical contacts $(100 \mu \mathrm{m} \times 100 \mu \mathrm{m})$ were patterned on top of $\mathrm{MoS}_{2}$ flakes using conventional lift-off technique. Ti $(10 \mathrm{~nm})$ and $\mathrm{Au}(300 \mathrm{~nm})$ were deposited by electron-beam evaporation at room temperature. The device was then annealed at $200^{\circ} \mathrm{C}$ in a vacuum tube furnace for $2 \mathrm{~h}\left(100 \mathrm{sccm} \mathrm{Ar}\right.$ and $\left.10 \mathrm{sccm} \mathrm{H}_{2}\right)$ to remove resist residue and to decrease contact resistance.

Measurements. The thickness of $\mathrm{MoS}_{2}$ was measured using an AFM (Nanoscope III, Digital Instruments-Veeco, USA). The electron backscatter diffraction (JEOL JSM7000F, Japan) and Raman spectra (Renishaw RM-1000 inVia, UK) with a 514-nm Ar laser were measured to characterize the $\mathrm{MoS}_{2}$ flakes on the substrate. Electrical characterizations were carried out with current-voltage measurements (Keithley, Semiconductor Characterization System 4200-SCS). During the lowtemperature measurement of transport properties, temperature was controlled using a variable temperature cryogenic probe system (LakeShore, TTPX). Capacitance-voltage characteristics were measured with an HP 4284A Precision LCR Metre.

\section{References}

1. Novoselov, K. S. et al. Two dimensional atomic crystals. Proc. Nat. Acad. Sci. 102, 10451-10453 (2005).

2. Frey, G. L. et al. Optical-absorption spectra of inorganic fullerenelike $\mathrm{MS}_{2}$ (M=Mo, W). Phys. Rev. B 57, 6666-6671 (1998).

3. Fivaz, R. \& Mooser, E. Mobility of charge carriers in semiconducting layer structures. Phys. Rev. 163, 743-755 (1967).

4. Mak, K. F. et al. Atomically thin $\mathrm{MoS}_{2}$ : a new direct-gap semiconductor Phys. Rev. Lett. 105, 136805 (2010).

5. Radisavljevic, B. et al. Single-layer $\mathrm{MoS}_{2}$ transistors. Nat. Nanotech. 6, 147-150 (2011).

6. Kamiya, T. et al. Present status of amorphous In-Ga-Zn-O thin-film transistors. Sci. Technol. Adv. Mater. 11, 044305 (2010).

7. Ayari, A. et al. Realization and electrical characterization of ultrathin crystals of layered transition-metal dichalcogenides. J. Appl. Phys. 101, 014507 (2007).

8. Podzorov, V. et al. High-mobility field-effect transistors based on transition metal dichalcogenides. Appl. Phys. Lett. 84, 3301-3303 (2004).

9. Meric, I. et al. Current saturation in zero-bandgap, top-gated graphene fieldeffect transistors. Nat. Nanotech. 3, 654-659 (2008).

10. Wilson, J. A. \& Yoffe, A. D. The transition metal dichalcogenides discussion and interpretation of the observed optical, electrical and structural properties. Adv. Phys. 18, 193-335 (1969).

11. Sze, S. M. Physics of Semiconductor Devices 446-448 2nd edn (Wiley, 1981).

12. Park, D. -G. et al. Characteristics of $n^{+}$polycrystalline-Si/ $\mathrm{Al}_{2} \mathrm{O}_{3} / \mathrm{Si}$ metaloxide- semiconductor structures prepared by atomic layer chemical vapor deposition using $\mathrm{Al}\left(\mathrm{CH}_{3}\right)_{3}$ and $\mathrm{H}_{2} \mathrm{O}$ vapor. J. Appl. Phys. 89, 6275-6280 (2001).

13. Wallace, R. M. et al. Atomic layer deposition of dielectrics on Ge and III-V materials for ultrahigh performance transistors. MRS Bull. 34, 493-503 (2009).
14. Natori, K. Ballistic metal-oxide-semiconductor field-effect transistor. J. Appl. Phys. 76, 4879-4890 (1994).

15. Zhang, Y. et al. Ambipolar $\mathrm{MoS}_{2}$ thin-flake transistors. Nano Lett. 12, 1136-1140 (2012).

16. Fivaz, R. Theory of layer structures. J. Phys. Chem. Solids 28, 839-845 (1967)

17. Han, S. W. et al. Band-gap transition induced by interlayer van der Waals interaction in $\mathrm{MoS}_{2}$. Phys. Rev. B. 84, 045409-045415 (2011).

18. Coehoorn, R. et al. Electronic structure of $\mathrm{MoSe}_{2}, \mathrm{MoS}_{2}$, and WSe 2 . I. Band-structure calculations and photoelectron spectroscopy. Phys. Rev. $B$ 35, 6195-6202 (1987)

19. Verble, J. L. \& Wieting, T. J. Lattice mode degeneracy in $\mathrm{MoS}_{2}$ and other layer compounds. Phys. Rev. Lett. 25, 362-365 (1970).

20. Gelmont, B. L. \& Shur, M. Polar optical-phonon scattering in three- and twodimensional electron gases. J. Appl. Phys. 77, 657-660 (1995).

21. Frindt, R. F. \& Yoffe, A. D. Physical properties of layer structures: optical and photoconductivity of thin crystals of molybdenum disulphide. Proc. Roy. Soc. A 273, 69-83 (1962)

22. Lee, C. et al. Anomalous lattice vibrations of single- and few layer $\mathrm{MoS}_{2}$. ACS Nano 4, 2695-2700 (2011).

23. de Heer, W. A. et al. Large area and structured epitaxial graphene produced by confinement controlled sublimation of silicon carbide. Proc. Nat. Acad. Sci. 108, 16900-16905 (2011).

24. Jena, D. \& Konar, A. Enhancement of carrier mobility in semiconducting nanostructures by dielectric engineering. Phys. Rev. Lett. 98, 136805-136809 (2007).

25. Konar, A. \& Jena, D. Tailoring the carrier mobility of semiconductor nanowires by remote dielectrics. J. Appl. Phys. 102, 123705-123708 (2007).

26. Liu, $\mathrm{H}$. \& Ye, P. MoS 2 dual-gate MOSFET with atomic-layer-deposited $\mathrm{Al}_{2} \mathrm{O}_{3}$ as top-gate dielectric. IEEE Electron Dev. Lett. 33, 546-548 (2012).

27. Konar, A. et al. Effect of high-k gate dielectrics on charge transport in graphene-based field-effect transistors. Phys. Rev. B 82, 115452 (2010).

\section{Acknowledgements}

We thank Dr. Eok Su Kim and Dr. Sunhee Lee of Samsung Electronics for their useful comments on the manuscript.

\section{Author contributions}

S.K. and W.C. initiated the research and worked on device fabrication, current-voltage measurements and analysis of $\mathrm{MoS}_{2}$ crystal properties. D.J. and A.K. performed device analysis and modelling, and calculations of scattering mechanisms. S.K., W.C., W.-S.H and J.L. performed capacitance-voltage measurements. J.H.L., C.J., J.-B.Y. and J.-Y.C worked on fabricating $\mathrm{MoS}_{2}$ flakes and atomic force microscopy measurements. J.Y. and H.K. worked on ALD. Y.W.J., S.Y.L. and K.K. advised on planning and executing the research. D.J., A.K., W.C. and S.K. wrote the manuscript.

\section{Additional information}

Supplementary Information accompanies this paper at http://www.nature.com/ naturecommunications

Competing financial interests: The authors declare no competing financial interests.

Reprints and permission information is available online at http://npg.nature.com/ reprintsandpermissions/

How to cite this article: Kim, S. et al. High-mobility and low-power thin-film transistors based on multilayer $\mathrm{MoS}_{2}$ crystals. Nat. Commun. 3:1011 doi: 10.1038/ncomms2018 (2012). 